\title{
Adolescents' Drawing and Divergent Thinking: Does Culture Matter?
}

\author{
Katherine Bottinelli ${ }^{1}$, Yena Kyeong ${ }^{1} \&$ Cecilia Cheung ${ }^{1}$ \\ ${ }^{1}$ Department of Psychology, University of California, Riverside, Riverside, United States \\ Correspondence: Cecilia Cheung, Department of Psychology, University of California, Riverside, CA 92521, \\ United States. Tel: 1-951-827-5266. E-mail: ccheung@ucr.edu
}

Received: August 19, 2018 Accepted: September 7, 2018 Online Published: September 11, 2018

doi:10.5539/jedp.v8n2p147

URL: http://doi.org/10.5539/jedp.v8n2p147

\begin{abstract}
The current study examined the association between adolescents' divergent thinking and features of their drawings in the United States and China. A total of 321 American $(n=125)$ and Chinese $(n=196)$ adolescents completed a battery of assessments on divergent thinking and free drawing adapted from the Torrance Tests of Creative Thinking (TTCT). Central (e.g., focal object) and contextual (e.g., background) features characterizing adolescents' drawings were coded. Results indicated that Chinese adolescents included more central features in their drawings compared to their American counterparts. Chinese, but not American, adolescents' inclusion of contextual features was positively associated with their divergent thinking. Findings suggest the potential for culture to influence adolescents' cognition, such that contextual features in drawings may be particularly conducive to the development of divergent thinking in cultures where contextual sensitivity is emphasized.
\end{abstract}

Keywords: divergent thinking, drawing features, culture, adolescence

\section{Introduction}

\subsection{Problem Statement}

Divergent thinking, defined as the ability to produce a number of unique ideas in response to a problem, has been considered as an important component in the development of children's creative outcomes (Ma, 2009; Runco, 1986; 2008). As a plethora of empirical evidence documents the conducive role of divergent thinking for children's academic success, factors that can cultivate children's divergent thinking have been of interest to researchers in education and psychology (Fleith, Renzulli, \& Westberg, 2002). Researchers argue that adolescence is an important stage marked by flexibility in cognition (Crone \& Dahl, 2012; Steinberg, 2005), rendering it an ideal period to understand the development of divergent thinking (Kleibeuker, De Dreu, \& Crone, 2013; 2016). To date, the link between divergent thinking and artistic expressions, such as drawings, is unclear. Adolescents' drawings are not only a form of creative expression, but also serve as a useful source to understand the development of creative thinking (Kim, 2006). Among a wide array of features characterizing adolescents' drawings, the current study aimed to investigate the role of central features (e.g., focal object) and contextual features (e.g., background objects) in adolescents' drawings in relation to their divergent thinking skills. In this context, we also examined whether the association between drawing features and creative thinking skills is dependent on culture.

\subsection{Divergent Thinking in Child Development}

Divergent thinking can be understood as a proxy for one's potential for creative problem solving (Runco, 1991). In the extant literature, divergent thinking has been indexed by fluency (the ability to produce many relevant ideas), flexibility (the ability to produce a wide variety of ideas), originality (the ability to produce novel and unique ideas), and elaboration (the ability to develop ideas in detail; Gajda, Karwowski, \& Beghetto, 2017; Guilford, 1967; Ma, 2009; Torrance, 1960). A large body of research has examined the role of divergent thinking in children's outcomes, including creative performance (e.g. writing, art, and music), academic achievement, and psychosocial functioning (Kim, 2008; Milgram \& Hong, 1993; Runco, 1986; Runco, Millar, Acar, \& Cramond, 2010). A study by Jeon, Moon, and French (2011) found that children who showed higher divergent thinking skills exceled in their creative performance in art compared to those with lower divergent thinking. In addition, research has consistently shown that divergent thinking is linked with children's school adjustment (e.g., Anwar \& Aness, 2012; Garaigordobil \& Berrueco, 2011), self-competence (e.g., Kolloff \& Feldhusen, 1984; Meador, 1995), and psychological well-being (e.g., Russ \& Kaugars, 2001; Russ \& Schafer, 2006). Notably, Baer (1994) examined the effects of divergent thinking training on creative activity among second graders and found that children who were exposed to divergent 
thinking training for several weeks showed considerable increase in performance in creative tasks (e.g., storytelling, story writing, poetry writing), compared to children in the control group.

Furthermore, longitudinal studies lend support to the relations between divergent thinking and creative achievement over time (Runco et al., 2010; Torrance, 1969). In a 50-year follow-up with participants from Torrance's seminal study on creative thinking, researchers found that divergent thinking scores in early childhood were associated with participants' creative achievement or personal accomplishment in creative activities (e.g., community outreach work, involvement in hobbies) in adulthood (Runco et al., 2010; Torrance 2002). Another longitudinal research also indicates that divergent thinking prowess in early childhood is significantly related to teachers' evaluations of creativity in pre-adolescence (Harrington, Block, \& Block, 1983).

\subsection{Predictors of Divergent Thinking}

Given the importance of divergent thinking skills in children's educational success, there has been much attention devoted to understanding the factors associated with its development (Batey \& Furnham, 2006; Batey, Furnham, \& Safiullina, 2010). Some researchers believe that intelligence and creativity are interrelated cognitive capabilities (Guilford, 1967). To evaluate this idea, studies have explored intelligence as a predictor of divergent thinking and found that high intelligence or above-average intelligence was predictive of divergent thinking (Barron \& Harrington, 1981; Batey \& Furnham, 2006; Guilford \& Christensen, 1973; Shi, Wang, Yang, Zhang, \& Xu, 2017). Beyond cognitive abilities, other research has focused on personality as a correlate of divergent thinking. Studies have demonstrated that divergent thinking is positively associated with Openness to Experience and Extraversion and negatively with Conscientiousness (Batey, Chamorro-Premuzic, \& Furnham, 2009; Furnham, Crump, Batey, \& Chamorro-Premuzic, 2009; McCrae, 1987). Other psychological factors, including emotions (Lieberman, 1977; Russ \& Schafer, 2006) and age, have also been found to correlate with divergent thinking (Kim, 2011).

\subsection{Linking Drawing and Thinking Skills}

Beyond cognitive and psychological factors, emerging research has focused on specific elements in drawings in relation to divergent thinking skills. The Torrance Tests of Creative Thinking (TTCT; Torrance, 1966) is a widely used measurement for divergent thinking (Gajda et al., 2017). Torrance developed the TTCT to analyze responses from specially designed verbal tasks and figural tasks for components of creativity (Torrance, 1960). While verbal tasks involve the use of written or oral language, figural tasks involve drawing. The figural test battery is comprised of three subtests, including Picture Construction, Picture Completion, and Repeated Figures. The TTCT figural tasks allow participants to draw freely, thereby relinquishing any boundaries that may be present in written assessments (Jellen \& Urban, 1989).

Although divergent thinking can be measured through figural tests in TTCT, the connection between cognitive skills and specific features in drawing is unclear. Herein, drawing features are defined as the characteristic of the product, which can include the complexity, placement of elements, and the attention to the relationships among the elements in the product. Generally, in assessing children's drawings, a variety of dimensions and characteristics have been utilized, including the number of figures, absolute size and proportions of the objects, complexity, and details (Cherney, Seiwert, Dickey, \& Flichtbeil, 2006; La Voy et al., 2001). Among a wide array of features characterizing drawings and visual arts, the inclusion of central and discrete objects (i.e., central features) and information about the context of objects and their relationship (i.e., contextual features) are two salient characteristics (Masuda, Gonzalez, Kwan, \& Nisbett, 2008). These features, as manifested in drawings and visual arts, can be used to evaluate important facets of an individual's cognitive processes, given that they may reflect distant attentional styles associated with cognition. Specifically, researchers have argued that the inclusion of central features can be indicative of a more analytic and object-oriented cognitive style; in contrast, the inclusion of contextual features may indicate more holistic and context-sensitive patterns of attention (Masuda et al., 2008).

While studies have examined the presence of central and contextual features in children's and adults' drawings (e.g., Masuda et al., 2008; Senzaki, Masuda, \& Nand, 2014), to our knowledge, there is no research focusing on their relations to divergent thinking. Despite this gap, research on relevant topics has provided important insights for this subject matter. For example, researchers have investigated the implications of cognitive and attentional styles for divergent thinking. Studies found that individuals with field-independent thinking styles (i.e., the tendency to perceptually separate an object from the context) show greater levels of divergent thinking, compared to individuals who have more field-dependent thinking styles (i.e., the tendency to perceive the field or context as a whole; e.g., Miller, 2007; Riding \& Cheema, 1991).

\subsection{Does Culture Matter?}

Children and adolescents' drawings reflect their representational understanding and knowledge about their 
surrounding environments (Cherney et al., 2006), which can often be influenced by cultural conventions (Masuda et al., 2008). As a form of cultural symbols, individuals' production of drawings is a useful tool to understand their cognition and thinking in relation to the norms in a given culture. Holistic (vs. analytic) patterns of attention as reflected in drawings of contextual (vs. focal) information have been a particularly relevant topic. The general contention is that different cultures foster different modes of information processing, including visual perception and selective attention (Kitayama, Duffy, Kawamura, \& Larsen, 2003). In line with this idea, emerging research indicates that individuals' attentional patterns are dependent on the cultural context in which they reside (Masuda $\&$ Nisbett, 2001). For example, when passing judgment on a focal object in visual perception tasks, Japanese individuals tend to incorporate more contextual information, while North Americans tend to pay less attention to contextual information (Kitayama et al., 2003).

Following the seminal work of Nisbett, Peng, Choi, and Norenzayan (2001) on the influence of culture on perception, research has documented cross-cultural differences in individuals' cognitive processing styles between Eastern (mostly East Asian countries) and Western cultures (mostly the United States), with growing neurobiological evidence augmenting the identified differences (Han et al., 2013; Hedden, Ketay, Aron, Markus, \& Gabrieli, 2008). Research indicates that Easterners typically have broader and more distributed attention and focus more on the relational context, while Westerners have more focused attention and exploit information about central objects and individuals in visual perception (e.g., Kitayama et al., 2003), recognition judgment (e.g., Masuda \& Nisbett, 2001), as well as emotional understanding and appraisal (e.g., Kuwabara, Son, \& Smith, 2011; Uchida, Townsend, Markus, \& Bergsieker, 2009). Moreover, this cultural variation in context-sensitivity is evident from early childhood (Imada, Carlson, Itakura, 2013; Kuwabara \& Smith, 2016), with such variations becoming more pronounced among older children (Senzaki et al., 2014).

As a wealth of evidence indicates cross-cultural differences in contextual sensitivity at perceptual levels, one can speculate that Easterners and Westerners may also differ in contextual sensitivity in their drawings. Contextual sensitivity in drawings is often assessed with the ratio of the location of the horizon to the entire landscape (Masuda et al., 2008). Consistent with findings on attentional patterns, studies using archival data of drawings and photographs indicate that East Asians tend to include more contextual information in visual images compared to their Western counterparts (e.g., Huang \& Park, 2013; Masuda et al., 2008). For example, when asked to draw a landscape including certain objects (e.g., house, tree, person, etc.), individuals from Western cultures focused more on salient objects, with a tendency to exclude background information in the images. However, East Asians focused more on objects that make up the context of the scene (e.g., clouds, additional buildings; Masuda et al., 2008). In a similar vein, Senzaki and colleagues (2014) analyzed Canadian and Japanese children's landscape drawings and collages. Findings from their research demonstrated that among children in $2^{\text {nd }}$ and higher grades, Japanese children included significantly more contextual information (e.g., vehicles, animals, plants) in their landscape collages, compared to their Canadian counterparts.

\subsection{The Current Study}

The goal of the current research was to examine the association between features of early adolescents' drawings and their divergent thinking. We focused on central vs. contextual features of the drawings produced by American and Chinese adolescents, given prior research on the distinct attentional styles characterizing Easterners and Westerners. We examined the link between drawings and divergent thinking among early adolescents, with a typical age range between 11 to 14 (Green, 1994; Oberle, Schonert-Reichl, Lawlor, \& Thomson, 2012), because this stage of development is characterized by high levels of cognitive flexibility (Crone \& Dahl, 2012; Steinberg, 2005). Moreover, adolescence is generally marked by cultural susceptibility (Choudhury, 2009), suggesting the importance of cross-cultural studies on the representations of cognitive styles reflected in drawings during this developmental phase. Based on past empirical evidence, we expected that American adolescents would include more central features in their drawings while Chinese adolescents would incorporate more contextual features. We also predicted that both central and contextual features would be associated with adolescents' divergent thinking. However, given that contextual sensitivity is more prevalent in East Asian cultural context, we predicted that Chinese (vs. American) adolescents' attention to contextual features would be more strongly associated with their divergent thinking.

\section{Method}

\subsection{Participants and Procedure}

Participants were 321 American ( $n=125 ; 76$ female and 49 male) and Chinese ( $n=196 ; 79$ female and 117 male) early adolescents, ranging from 11 to 14 years of age. Adolescents were recruited from four middle schools and one summer program in the United States and five middle schools in China. Using an opt-in procedure, 
participants were required to have their parents complete a parental consent form and personally sign an assent form to participate in the study. After the parental consent and child assent forms were returned, participants completed surveys and an assessment on divergent thinking during a 1-hour session in their classrooms or the school library. Trained native researchers were present to provide verbal instructions and answer questions. Participants were informed that they could withdraw from the study or skip over any questions without any negative consequences. Participants completed the survey and assessment privately. All finished work was placed in sealed envelopes to ensure confidentiality. The American participants recruited from the summer program completed the survey at home and returned the packets to researchers by mail, or completed an online version of the survey through Qualtrics ${ }^{\circledR}$. For each survey completed, participants received a $\$ 5$ gift card as a token of appreciation.

\subsection{Measures}

\subsubsection{Divergent Thinking Assessment}

Divergent thinking was measured using two multiple uses tasks adapted from the TTCT (Guilford, 1967; Torrance, 1972). Participants engaged in abstract thinking tasks regarding an object's number of possible functions (e.g., name as many uses of a mirror as possible). The question prompts for the two multiple uses tasks were: "List as many uses of a mirror as you can" and "List as many uses for a newspaper as you can".

The responses were scored for divergent thinking using a five category system that followed Torrance's test manual (Torrance, 1966), which included frequency (the number of relevant responses), elaborateness (the number of responses that had additional details), practicality (the number of responses that illustrated feasible uses of the object), inventiveness (the number of responses that involved creating something new from the object), and unintended use (the number of responses that reflected new ways of using the object). The coding scheme was translated and back-translated to ensure cross-cultural validity. Additionally, $5 \%$ of the data from each country was coded by a bicultural coder to ensure that the coding system was used similarly in the two countries. Coder training in the United States and China consisted of two parts. In the first phase of the training, coders were presented with definitions and examples of each coding category. In the second phase, coders were given twenty samples to practice. Coders were retrained on the practice samples until they reached at least $80 \%$ agreement.

All categories were coded on the number of valid creative responses. A response was considered valid if it corresponded to one of the definitions in the coded categories. For example, the fluency category asks for valid responses corresponding to the definition of "ability to produce a relevant response". In the case of the newspaper prompt, a coder assessing responses for fluency would code "make a paper airplane" as a valid response and "New York Times" as an invalid response.

\subsubsection{Drawing Features}

To assess adolescents' drawings, figural forms of the TTCT were administered, and features characterizing participants' drawing were assessed. Two types of drawing tasks (Picture Construction and Repeated Figures) were given to the participants. For the purpose of the current study, we focused only on responses from the Repeated Figures tasks. In this task, participants were instructed to draw freely on a paper that contained seven circles and was otherwise blank. For the task, approximately $95 \%$ of the paper was left blank. The task was not timed such that participants could spend as much or as little time as they wished to complete their drawing.

From the drawing task, central and contextual features were analyzed. The coding procedure used for drawing features was similar to the coding procedure used for the divergent thinking assessment. The coding scheme was translated and back-translated to ensure validity, followed by a two-part training for the coders in both countries. The first phase of training consisted of a presentation on definitions and examples of each coding category. In the second phase, coders were given twenty samples to practice until they reached at least $80 \%$ agreement. After coders completed the two-part training, they worked in pairs to complete the coding procedure.

Central features were defined as the number of focal objects, while contextual features were defined as the number of background objects. Higher scores for central features indicate that the participants utilized more focal elements to complete the picture. For example, if participants completed a picture of the circles as candies in a bag, they would receive points for including focal elements, as the candies act as central features in the picture. Alternatively, higher scores on contextual features indicate that the participant utilized more background elements to complete the picture. For example, if a participant completed a picture of circles as all balloons, with one person holding all the balloons, the participant would receive a point for background elements as the balloons act as contextual features in the image. 


\section{Results}

Two sets of analyses were conducted to address the research questions. First, independent samples $t$-tests were used to evaluate differences in drawing features and divergent thinking between American and Chinese adolescents. Second, a series of hierarchical regression analyses was employed to examine the associations between drawing features and divergent thinking, with attention to between-country differences.

\subsection{Descriptive Statistics and Zero-Order Correlations}

Table 1 presents the descriptive statistics for the main study variables. With regard to drawing features, independent samples $t$-tests revealed that Chinese adolescents included more central features in their drawings, compared to American adolescents, $t(231)=-2.83, p<.01$. However, this was not the case for contextual features, where the levels of such features were similar in the two countries, $t(319)=0.79, n s$. For scores on divergent thinking, there were significant differences in the raw scores of each of the five subcategories between the two countries: American adolescents scored higher on fluency, practicality, and unintended use, $t(186)>5.42, p<.001$, while Chinese adolescents scored higher on elaborateness and inventiveness, $t(300)>-5.67, p<.001$. When collapsed across the five dimensions of divergent thinking, American adolescents scored higher $(M=5.58, S D=$ 3.36) than their Chinese counterparts $(M=4.29, S D=3.16), t(340)=3.63, p<.001$. For the purpose of the current study, a combined score for divergent thinking was created by first standardizing each dimension of divergent thinking within each country and then taking an average across the five standardized dimensions.

Table 1. Descriptive statistics for main study variables

\begin{tabular}{|c|c|c|c|c|c|}
\hline \multirow[b]{2}{*}{ Variable } & \multicolumn{2}{|c|}{ US $(n=149)$} & \multicolumn{2}{|c|}{ China $(n=193)$} & \multirow[b]{2}{*}{$t$} \\
\hline & $M$ & $S D$ & $M$ & $S D$ & \\
\hline \multicolumn{6}{|l|}{ Drawings } \\
\hline 1. Central features & 5.14 & 2.22 & 5.82 & 1.87 & $-2.83^{* *}$ \\
\hline 2. Contextual features & 0.52 & 1.34 & 0.32 & 2.57 & 0.79 \\
\hline 3. Frequency of drawings & 5.71 & 1.82 & 6.15 & 2.85 & -1.54 \\
\hline \multicolumn{6}{|l|}{ Divergent Thinking } \\
\hline \multicolumn{6}{|l|}{ Subcategories } \\
\hline 1. Fluency & 10.20 & 5.48 & 7.21 & 4.47 & $5.42^{* * *}$ \\
\hline 2. Elaborateness & 0.56 & 2.01 & 2.40 & 2.90 & $-6.97^{* * *}$ \\
\hline 3. Practicality & 10.13 & 5.47 & 6.85 & 4.23 & $6.06^{* * *}$ \\
\hline 4. Inventiveness & 1.96 & 1.98 & 3.79 & 3.90 & $-5.67^{* * *}$ \\
\hline 5. Unintended use & 5.03 & 4.62 & 1.20 & 1.88 & $9.51^{* * *}$ \\
\hline
\end{tabular}

Note. ${ }^{* *} p<.01,{ }^{* * *} p<.001$.

Before formally testing the central hypotheses, zero-order correlation analyses were conducted to examine the associations among the key study variables. As shown in Table 2, in both the United States and China, central features and contextual features were negatively correlated, $r=-.56, p<.01$ for the United States and $r=-.20, p$ $<.05$ for China. In the United States, the association between divergent thinking and central and contextual features did not reach statistical significance, $r \mathrm{~s}=-.02, n s$. In China, however, divergent thinking was negatively correlated with central features, $r=-.17, p<.05$, and positively correlated with contextual features $r=.28, p<.01$. 
Table 2. Associations between drawing features and divergent thinking

\begin{tabular}{llll}
\hline & 1 & 2 & 3 \\
\hline 1. Central Features & - & $-.20^{*}$ & $-.17^{*}$ \\
2. Contextual Features & $-.56^{* *}$ & - & $.28^{* *}$ \\
3. Divergent Thinking & -.02 & -.02 & - \\
\hline
\end{tabular}

Note. Correlations for the American sample are presented below the diagonal and correlations for the Chinese sample are presented above the diagonal. ${ }^{*} p<.05 .{ }^{* *} p<.01$.

\subsection{Drawing Features and Divergent Thinking}

Hierarchical regression analyses were conducted to evaluate whether the strength of the associations between drawing features and divergent thinking differed between the two countries. Two separate models were evaluatedone for central and the other for contextual features. To ensure that the findings were not simply due to the sheer number of drawing features and possible gender differences, all models included the total number of drawing features, as well as gender ( $1=$ male, $2=$ female $)$ as covariates in Step 1. In Step 2, the dummy coded variable of country $(0=$ the United States, $1=$ China $)$ and central or contextual features were included. To evaluate the extent to which the associations were similar in the two countries, Step 3 included a two-way interaction term for country and central or contextual features.

\subsubsection{Central Features}

Table 3 presents the results from the hierarchical regression analysis. The total number of drawing features was not significantly associated with divergent thinking, $\beta=.10, n s$. However, central features in adolescents' drawings were negatively associated with their divergent thinking, $\beta=-.23, p<.01$, and the strength of the association was similar in the United States and China, $\beta=-.30, p=.10$.

Table 3. Results from regression predicting divergent thinking from central features

\begin{tabular}{|c|c|c|c|c|}
\hline Variables & $B$ & $S E(B)$ & $\beta$ & $\Delta R^{2}$ \\
\hline \multicolumn{5}{|l|}{ Step 1} \\
\hline Gender $(1=$ Male, 2 = Female $)$ & -.02 & .10 & -.01 & \\
\hline Frequency of Circle Drawing & .04 & .02 & $.10^{\dagger}$ & \\
\hline \multicolumn{5}{|l|}{ Step 2} \\
\hline Gender $(1=$ Male, 2 = Female $)$ & -.00 & .10 & -.00 & $.039^{* *}$ \\
\hline Frequency of Circle Drawing & .08 & .02 & $.22^{* *}$ & \\
\hline Country $(0=$ US, $1=$ China $)$ & .12 & .10 & .07 & \\
\hline Central Features & -.10 & .03 & $-.23^{* *}$ & \\
\hline \multicolumn{5}{|l|}{ Step 3} \\
\hline Gender $(1=$ Male, 2 = Female $)$ & .01 & .10 & .00 & $.009^{\dagger}$ \\
\hline Frequency of Circle Drawing & .08 & .02 & $.23^{* *}$ & \\
\hline Country $(0=\mathrm{US}, 1=$ China $)$ & .56 & .28 & $.32^{*}$ & \\
\hline Central Features & -.06 & .04 & -.13 & \\
\hline Country $\times$ Central Features & -.08 & .05 & $-.30^{\dagger}$ & \\
\hline
\end{tabular}

Note. ${ }^{\dagger} p<.10 .{ }^{*} p<.05 .{ }^{* *} p<.01$.

\subsubsection{Contextual Features}

Results from the hierarchical regression analysis predicting divergent thinking from country and contextual features are presented in Table 4. As with the previous analysis, the total number of drawing features was not significantly associated with divergent thinking, $\beta=.10, n s$. However, contextual features were positively 
associated with divergent thinking, $\beta=.25, p<.01$. This association was qualified by a significant interaction between country and contextual features, $\beta=.34, p<.05$. The interaction effect was probed by a series of simple slope analyses. As shown in Figure 1, although there was a negative association between American adolescents' use of contextual features and divergent thinking, such a trend did not reach statistical significance, $\beta=-.03, t=$ $-.03, n s$. However, in line with our expectation, the association was significant in China, $\beta=.23, t=4.14, p<.001$, such that the more Chinese adolescents included contextual features in their drawings, the higher were their scores on divergent thinking.

Table 4. Results from regression predicting divergent thinking from contextual features

\begin{tabular}{|c|c|c|c|c|}
\hline Variables & $B$ & $S E(B)$ & $\beta$ & $\Delta R^{2}$ \\
\hline \multicolumn{5}{|l|}{ Step 1} \\
\hline Gender $(1=$ Male, $2=$ Female $)$ & -.02 & .10 & -.01 & \\
\hline Frequency of Circle Drawing & .04 & .02 & $.10^{\dagger}$ & \\
\hline \multicolumn{5}{|l|}{ Step 2} \\
\hline Gender $(1=$ Male, $2=$ Female $)$ & .00 & .10 & .00 & $.038^{* *}$ \\
\hline Frequency of Circle Drawing & -.02 & .03 & -.06 & \\
\hline Country $(0=$ US, $1=$ China $)$ & .12 & .10 & .07 & \\
\hline Contextual Features & .10 & .03 & $.25^{* *}$ & \\
\hline \multicolumn{5}{|l|}{ Step 3} \\
\hline Gender $(1=$ Male, $2=$ Female $)$ & .01 & .10 & .01 & $.015^{*}$ \\
\hline Frequency of Circle Drawing & -.04 & .03 & -.12 & \\
\hline Country $(0=$ US, $1=$ China $)$ & .06 & .10 & .04 & \\
\hline Contextual Features & -.01 & .06 & -.03 & \\
\hline Country $\times$ Contextual Features & .14 & .06 & $.34^{*}$ & \\
\hline
\end{tabular}

Note. ${ }^{\dagger} p<.10 .{ }^{*} p<.05 .{ }^{* *} p<.01$.

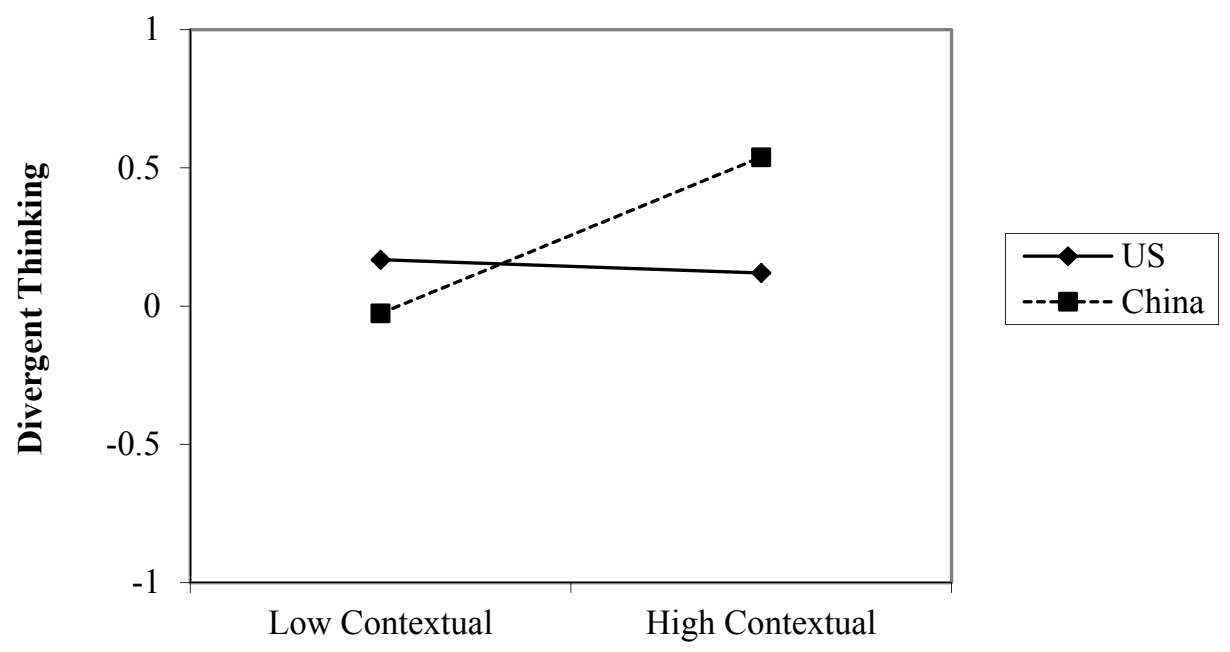

Figure 1. Interaction effect between country and contextual features

\section{Discussion}

The current study aimed to examine the associations between divergent thinking and drawing features (central and contextual features) among adolescents in the United States and China. Findings indicated that Chinese adolescents included more central features in their drawings compared to American adolescents, while the extent 
of contextual features used in drawings did not differ between the two countries. Consistent with our predictions, Chinese adolescents' inclusion of contextual features in their drawings was positively associated with their divergent thinking; however, this was not the case for adolescents in the United States.

Previous research has identified that East Asians tend to include more contextual information in their visual images in comparison to their Western counterparts (Kitayama et al., 2003; Masuda et al., 2008), as Westerners typically pay more attention to central objects and exclude background information in their visual and artistic presentations (Kuwabara et al., 2011; Masuda et al., 2008; Masuda \& Nisbett, 2001; Senzaki et al., 2014). Contrary to these findings among elementary school-aged children and adults, the current research found that Chinese adolescents utilized more central features in their drawings compared to American adolescents. Additionally, the findings indicated no difference in the number of contextual features included in the drawings among adolescents in the United States and China. The discrepancy between the current and prior findings may be attributed to participants' age and their varying degree of internalization of cultural socialization. Extending the work of Senzaki and colleagues (2014), Nand, Masuda, Senzaki, and Ishii (2014) demonstrated that cultural variability in the drawing features between Japanese and Canadian may vary across the developmental stage, such that cultural differences in aspects of drawing were found in elementary school children and young adults, while cultural similarities were evident in adolescents. Nand and colleagues (2014) speculate that young children may seek to internalize dominant cultural norms but become prone to seek alternative values as they enter adolescence, and adults may resume the culturally normative behavioral patterns. In line with this idea, it is possible that Chinese (vs. American) adolescents in the current study scored higher on central features because the effect of cultural socialization of sensitivity to context may not be as salient during this stage of development, thereby minimizing variations among adolescents' expression across cultures (Kuwabara \& Smith, 2016; Nand et al., 2014).

Alternatively, the discrepancy may be due to the nature of the drawing task used in the current study. In the current study, adolescents' drawing was guided by a prompt (i.e., circles) on the paper, as opposed to free drawing on a blank paper. Although researchers argue that the figural forms of the TTCT are a fair assessment of divergent thinking across cultural contexts (for a review, see Kim, 2006), it is unclear whether the prompt elicited different levels of contextual sensitivity between American and Chinese adolescents, thereby introducing an extraneous factor that may influence American and Chinese adolescents' inclusion of central features in their drawings.

The current research found that Chinese (vs. American) adolescents' attention to contextual features was more strongly associated with their divergent thinking. This finding is consistent with much prior research indicating differences between Easterners and Westerners' contextual sensitivity (Kitayama et al., 2003; Senzaki et al., 2014). Given that divergent thinking pertains to individuals' capabilities to generate ideas beyond the boundaries of their own culture (Leung \& Chiu, 2008), one may presume that deviation from cultural conventions would facilitate creative thinking. However, theorists postulate that the definition and value of creativity may differ across cultures (Niu \& Sternberg, 2002) and that creativity in East Asian contexts can coexist with the desire to conform (Dineen $\& \mathrm{Niu}, 2008)$.

With regard to central features, this research found that the inclusion of central features was associated with lower levels of divergent thinking among adolescents in both countries. This finding may appear surprising at first blush, however, preliminary support for this negative association can be found in the literature on thinking styles. For example, Zhang (2002) found that creative thinking was positively associated with holistic thinking style (i.e., processing information in a synthesized manner) and negatively with analytic thinking style (i.e., processing information in a piecemeal manner) among Chinese college students. Although more research among adolescents is warranted, our findings suggest that the inclusion of central (vs. contextual) features may hinder adolescents' ability to generate novel uses for objects.

Taken together, the current research provides novel evidence on the relationship between divergent thinking and features of early adolescents' drawings across cultural contexts. Prior research has focused on the benefits of fostering divergent thinking among school-age children to increase their creative outcomes academically (e.g. Kim, 2008; Runco, 1986), artistically (e.g. Baer, 1994; Milgram \& Hong, 1993), and longitudinally (e.g. Runco et al., 2010). Building on this body of work, the current study provides preliminary evidence on how drawing features can be relevant to adolescents' divergent thinking. Findings may shed light on the design of culturally sensitive educational programs to support the development of divergent thinking skills.

\subsection{Limitations and Future Directions}

The current study has several limitations, which offer important directions for further research in this area. First, the samples in the current research were drawn from specific areas in the United States and China and did not fully reflect the two countries' demographic variability. While nationality was used as a proxy of culture, it is important 
to note that micro-cultures are often prevalent within a country. Indeed, the United States is an ethnically diverse country such that cognitive processes may differ among adolescents from distinct ethnic subgroups (Bandura, 2002). Future studies should consider potential within-country variability by including samples from several regions within a country as well as across a wider array of Eastern and Western countries.

Another limitation of the current research pertains to the use of a single measure to assess divergent thinking. We used the verbal task in TTCT to gauge adolescents' divergent thinking given that it is one of the most widely used assessments and is valid across cultures (Kim, 2006). Despite the widespread use of TTCT, researchers have debated the validity of using a single assessment to measure divergent thinking, given that creativity is a complex phenomenon and may be better understood through the use of multiple testing approaches (Johnson \& Fishkin, 1999; Kim, 2006). Future studies may consider using additional methods, such as evaluations by independent judges or teachers, to assess adolescents' divergent thinking skills.

Third, this research utilized a cross-sectional design, which fails to capture potential changes in adolescents' divergent thinking over time. Past research has shown that children's creative potential may decline as they grow older (Imada et al., 2013). Hence, it is important to understand how divergent thinking and the expression of drawing features change over the course of development. As Nand et al. (2014) elucidated the different patterns of internalization of cultural socialization in developmental stages, it would be informative to investigate whether the associations between expression of drawing features and divergent thinking are similar among American and Chinese children before they enter adolescence.

\subsection{Conclusions}

The current study provides an emerging avenue for research on divergent thinking by introducing the role of central and contextual features in drawings as correlates of adolescents' creative thinking skills. Findings support the notion that culture can shape adolescents' cognitive style, insofar as American and Chinese adolescents embedded distinct levels of contextual features in their drawings. Additionally, the inclusion of central drawing features was associated with adolescents' dampened divergent thinking, while contextual features were associated with higher levels of divergent thinking among Chinese but not American adolescents. Results suggest that contextual features may be particularly pertinent to divergent thinking among adolescents in cultures where contextual sensitivity is emphasized.

\section{References}

Anwar, M. N., \& Aness, M. (2012). An examination of the relationship between creative thinking and academic achievements of secondary school students. International Interdisciplinary Journal of Education, 1, 1-4.

Baer, J. (1994). Divergent thinking is not a general trait: A multidomain training experiment. Creativity Research Journal, 7, 35-46. https://doi.org/10.1080/10400419409534507

Bandura, A. (2002). Social cognitive theory in cultural context. Applied Psychology, 51, 269-290. https://doi.org/10.1111/1464-0597.00092

Barron, F., \& Harrington, D. M. (1981). Creativity, intelligence, and personality. Annual Review of Psychology, 32, 439-476. https://doi.org/10.1146/annurev.ps.32.020181.002255

Batey, M., Chamorro-Premuzic, T., \& Furnham, A. (2009). Intelligence and personality as predictors of divergent thinking: The role of general, fluid and crystallised intelligence. Thinking Skills and Creativity, 4, 60-69. https://doi.org/10.1016/j.tsc.2009.01.002

Batey, M., \& Furnham, A. (2006). Creativity, intelligence, and personality: A critical review of the scattered literature. Genetic, Social, and General Psychology Monographs, 132, 355-429. https://doi.org/10.3200/MONO.132.4.355-430

Batey, M., Furnham, A., \& Safiullina, X. (2010). Intelligence, general knowledge and personality as predictors of creativity. Learning and Individual Differences, 20, 532-535. https://doi.org/10.1016/j.lindif.2010.04.008

Cherney, I. D., Seiwert, C. S., Dickey, T. M., \& Flichtbeil, J. D. (2006). Children's drawings: A mirror to their minds. Educational Psychology, 26, 127-142. https://doi.org/10.1080/01443410500344167

Choudhury, S. (2009). Culturing the adolescent brain: What can neuroscience learn from anthropology? Social Cognitive and Affective Neuroscience, 5, 159-167. https://doi.org/10.1093/scan/nsp030

Crone, E. A., \& Dahl, R. E. (2012). Understanding adolescence as a period of social-affective engagement and goal flexibility. Nature Reviews Neuroscience, 13, 636-650. https://doi.org/10.1038/nrn3313

Dineen, R., \& Niu, W. (2008). The effectiveness of western creative teaching methods in China: An action research 
project. Psychology of Aesthetics, Creativity, and the Arts, 2, $42-52$. https://doi.org/10.1037/1931-3896.2.1.42

Fleith, D. D. S., Renzulli, J. S., \& Westberg, K. L. (2002). Effects of a creativity training program on divergent thinking abilities and self-concept in monolingual and bilingual classrooms. Creativity Research Journal, 14, 373-386. https://doi.org/10.1207/S15326934CRJ1434_8

Furnham, A., Crump, J., Batey, M., \& Chamorro-Premuzic, T. (2009). Personality and ability predictors of the "consequences" test of divergent thinking in a large non-student sample. Personality and Individual Differences, 46, 536-540. https://doi.org/10.1016/j.paid.2008.12.007

Gajda, A., Karwowski, M., \& Beghetto, R. A. (2017). Creativity and academic achievement: A meta-analysis. Journal of Educational Psychology, 109, 269-299. https://doi.org/10.1037/edu0000133

Garaigordobil, M., \& Berrueco, L. (2011). Effects of a play program on creative thinking of preschool children. The Spanish Journal of Psychology, 14, 608-618. https://doi.org/10.5209/rev_SJOP.2011.v14.n2.9

Green, M. (1994). Bright futures: Guidelines for health supervision of infants, children, and adolescents. McLean, VA: American Academy of Pediatrics.

Guilford, J. P. (1967). The nature of human intelligence. New York, NY: McGraw-Hill.

Guilford, J. P., \& Christensen, P. R. (1973). The one - way relation between creative potential and IQ. The Journal of Creative Behavior, 7, 247-252. https://doi.org/10.1002/j.2162-6057.1973.tb01096.x

Han, S., Northoff, G., Vogeley, K., Wexler, B. E., Kitayama, S., \& Varnum, M. E. (2013). A cultural neuroscience approach to the biosocial nature of the human brain. Annual Review of Psychology, 64, 335-359. https://doi.org/10.1146/annurev-psych-071112-054629

Harrington, D. M., Block, J., \& Block, J. H. (1983). Predicting creativity in preadolescence from divergent thinking in early childhood. Journal of Personality and Social Psychology, 45, 609-623. https://doi.org/10.1037/0022-3514.45.3.609

Hedden, T., Ketay, S., Aron, A., Markus, H. R., \& Gabrieli, J. D. (2008). Cultural influences on neural substrates of attentional control. Psychological Science, 19, 12-17. https://doi.org/10.1111/j.1467-9280.2008.02038.x

Huang, C. M., \& Park, D. (2013). Cultural influences on Facebook photographs. International Journal of Psychology, 48, 334-343. https://doi.org/10.1080/00207594.2011.649285

Imada, T., Carlson, S. M., \& Itakura, S. (2013). East-West cultural differences in context - sensitivity are evident in early childhood. Developmental Science, 16, 198-208. https://doi.org/10.1111/desc.12016

Jellen, H. G., \& Urban, K. K. (1989). Assessing creative potential world-wide: The first cross-cultural application of the Test for Creative Thinking-Drawing Production (TCT-DP). Gifted Education International, 6, 78-86. https://doi.org/10.1177/026142948900600204

Jeon, K. N., Moon, S. M., \& French, B. (2011). Differential effects of divergent thinking, domain knowledge, and interest on creative performance in art and math. Creativity Research Journal, 23, 60-71. https://doi.org/10.1080/10400419.2011.545750

Johnson, A. S., \& Fishkin, A. S. (1999). Assessment of cognitive and affective behaviors related to creativity. In A. S. Fishkin, B. Cramond, \& P. Olszewski-Kubilius (Eds.), Investigating creativity in youth: Research and methods (pp. 265-306). Cresskill, NJ: Hampton.

Kim, K. H. (2006). Can we trust creativity tests? A review of the Torrance Tests of Creative Thinking (TTCT). Creativity Research Journal, 18, 3-14. https://doi.org/10.1207/s15326934crj1801_2

Kim, K. H. (2008). Meta-analyses of the relationship of creative achievement to both IQ and divergent thinking test scores. The Journal of Creative Behavior, 42, 106-130. https://doi.org/10.1002/j.2162-6057.2008.tb01290.x

Kim, K. H. (2011). The creativity crisis: The decrease in creative thinking scores on the Torrance Tests of Creative Thinking. Creativity Research Journal, 23, 285-295. https://doi.org/10.1080/10400419.2011.627805

Kitayama, S., Duffy, S., Kawamura, T., \& Larsen, J. T. (2003). Perceiving an object and its context in different cultures: A cultural look at new look. Psychological Science, 14, 201-206. https://doi.org/10.1111/1467-9280.02432

Kleibeuker, S. W., De Dreu, C. K. W, \& Crone, E. A. (2013). The development of creative cognition across 
adolescence: Distinct trajectories for insight and divergent thinking. Developmental Science, 16, 2-12. https://doi.org/10.1111/j.1467-7687.2012.01176.x

Kleibeuker, S. W., De Dreu, C. K. W., \& Crone, E. A. (2016). Creativity development in adolescence: Insight from behavior, brain, and training studies. New Directions for Child and Adolescent Development, 151, 73-84. https://doi.org/10.1002/cad.20148

Kolloff, P. B., \& Feldhusen, J. F. (1984). The effects of enrichment on self-concept and creative thinking. Gifted Child Quarterly, 28, 53-57. https://doi.org/10.1177/001698628402800202

Kuwabara, M., \& Smith, L. B. (2016). Cultural differences in visual object recognition in 3-year-old children. Journal of Experimental Child Psychology, 147, 22-38. https://doi.org/10.1016/j.jecp.2016.02.006

Kuwabara, M., Son, J. Y., \& Smith, L. B. (2011). Attention to context: US and Japanese children's emotional judgments. Journal of Cognition and Development, 12, 502-517. https://doi.org/10.1080/15248372.2011.554927

La Voy, S. K., Pedersen, W. C., Reitz, J. M., Brauch, A. A., Luxenberg, T. M., \& Nofsinger, C. C. (2001). Children's drawings: A cross-cultural analysis from Japan and the United States. School Psychology International, 22, 53-63. https://doi.org/10.1177/0143034301221005

Leung, A. K. Y., \& Chiu, C. Y. (2008). Interactive effects of multicultural experiences and openness to experience on creative potential. Creativity Research Journal, 20, 376-382. https://doi.org/10.1080/10400410802391371

Lieberman, J. N. (1977). Playfulness: Its relationship to imagination and creativity. New York, NY: Academic Press.

Ma, H. H. (2009). The effect size of variables associated with creativity: A meta-analysis. Creativity Research Journal, 21, 30-42. https://doi.org/10.1080/10400410802633400

Masuda, T., Gonzalez, R., Kwan, L., \& Nisbett, R. E. (2008). Culture and aesthetic preference: Comparing the attention to context of East Asians and Americans. Personality and Social Psychology Bulletin, 34, 1260-1275. https://doi.org/10.1177/0146167208320555

Masuda, T., \& Nisbett, R. E. (2001). Attending holistically versus analytically: comparing the context sensitivity of Japanese and Americans. Journal of Personality and Social Psychology, 81, 922-934. https://doi.org/10.1037/0022-3514.81.5.922

McCrae, R. R. (1987). Creativity, divergent thinking, and openness to experience. Journal of Personality and Social Psychology, 52, 1258. https://doi.org/10.1037/0022-3514.52.6.1258

Meador, K. S. (1995). The effect of synectics training on gifted and nongifted kindergarten students. Talents and Gifts, 18, 55-73. https://doi.org/10.1177/016235329401800105

Milgram, R. M., \& Hong, E. (1993). Creative thinking and creative performance in adolescents as predictors of creative attainments in adults: A follow - up study after 18 years. Roeper Review, 15, 135-139. https://doi.org/10.1080/02783199309553487

Miller, A. L. (2007). Creativity and cognitive style: The relationship between field-dependence-independence, expected evaluation, and creative performance. Psychology of Aesthetics, Creativity, and the Arts, 1, 243-246. https://doi.org/10.1037/1931-3896.1.4.243

Nand, K., Masuda, T., Senzaki, S., \& Ishii, K. (2014). Examining cultural drifts in artworks through development and history: Cultural comparisons between Japanese and Western landscape paintings and drawings. Frontiers in Psychology: Cultural Psychology, 5. https://doi.org/10.3389/fpsyg.2014.01041.

Nisbett, R. E., Peng, K., Choi, I., \& Norenzayan, A. (2001). Culture and systems of thought: holistic versus analytic cognition. Psychological Review, 108, 291-310. https://doi.org/10.1037/0033-295X.108.2.291

Niu, W., \& Sternberg, R. (2002). Contemporary studies on the concept of creativity: The East and the West. The Journal of Creative Behavior, 36, 269-288. https://doi.org/10.1002/j.2162-6057.2002.tb01069.x

Oberle, E., Schonert-Reichl, K. A., Lawlor, M. S., \& Thomson, K. C. (2012). Mindfulness and inhibitory control in early adolescence. The Journal of Early Adolescence, 32, 565-588. https://doi.org/10.1177/0272431611403741

Riding, R., \& Cheema, I. (1991). Cognitive styles - an overview and integration. Educational Psychology, 11, 193-215. https://doi.org/10.1080/0144341910110301 
Runco, M. A. (1986). Divergent thinking and creative performance in gifted and nongifted children. Educational and Psychological Measurement, 46, 375-384. https://doi.org/10.1177/001316448604600211

Runco, M. A. (1991). The evaluative, valuative, and divergent thinking of children. The Journal of Creative Behavior, 25, 311-319. https://doi.org/10.1002/j.2162-6057.1991.tb01143.x

Runco, M. A. (2008). Commentary: Divergent thinking is not synonymous with creativity. Psychology of Aesthetics, Creativity, and the Arts, 2, 93-96. https://doi.org/10.1037/1931-3896.2.2.93

Runco, M. A., Millar, G., Acar, S., \& Cramond, B. (2010). Torrance Tests of Creative Thinking as predictors of personal and public achievement: A fifty-year follow-up. Creativity Research Journal, 22, 361-368. https://doi.org/10.1080/10400419.2010.523393

Russ, S. W., \& Kaugars, A. S. (2001). Emotion in children's play and creative problem solving. Creativity Research Journal, 13, 211-219. https://doi.org/10.1207/S15326934CRJ1302_8

Russ, S. W., \& Schafer, E. D. (2006). Affect in fantasy play, emotion in memories, and divergent thinking. Creativity Research Journal, 18, 347-354. https://doi.org/10.1207/s15326934crj1803_9

Senzaki, S., Masuda, T., \& Nand, K. (2014). Holistic versus analytic expressions in artworks: Cross-cultural differences and similarities in drawings and collages by Canadian and Japanese school-age children. Journal of Cross-Cultural Psychology, 45, 1297-1316. https://doi.org/10.1177/0022022114537704

Shi, B., Wang, L., Yang, J., Zhang, M., \& Xu, L. (2017). Relationship between divergent thinking and intelligence: An empirical study of the Threshold Hypothesis with Chinese children. Frontiers in Psychology, 8. https://doi.org/10.3389/fpsyg.2017.00254

Steinberg, L. (2005). Cognitive and affective development in adolescence. Trends in Cognitive Sciences, 9, 69-74. https://doi.org/10.1016/j.tics.2004.12.005

Torrance, E. P. (1960). Explorations in creative thinking. Education, 81, 216-220.

Torrance, E. P. (1966). The Torrance Tests of Creative Thinking-Norms-Technical Manual Research Edition-Verbal Tests, Forms A and B-Figural Tests, Forms A and B. Princeton, NJ: Personnel Press.

Torrance, E. P. (1969). Prediction of adult creative achievement among high school seniors. Gifted Child Quarterly, 13, 223-229. https://doi.org/10.1177/001698626901300401

Torrance, E. (1972). Can we teach children to think creatively? The Journal of Creative Behavior, 6, 114-143. https://doi.org/10.1002/j.2162-6057.1972.tb00923.x

Uchida, Y., Townsend, S. S., Markus, H. R., \& Bergsieker, H. B. (2009). Emotions as within or between people? Cultural variation in lay theories of emotion expression and inference. Personality and Social Psychology Bulletin, 35, 1427-1439. https://doi.org/10.1177/0146167209347322

Zhang, L. F. (2002). Thinking styles: Their relationships with modes of thinking and academic performance. Educational Psychology, 22, 331-348. https://doi.org/10.1080/014434102201385

\section{Copyrights}

Copyright for this article is retained by the author(s), with first publication rights granted to the journal.

This is an open-access article distributed under the terms and conditions of the Creative Commons Attribution license (http://creativecommons.org/licenses/by/4.0/). 